\title{
Compensated Tunable Capacitive Thin Film Hydrogen Sensor
}

\author{
D. J. Kirby, J. J. Zinck, D. T. Chang, F. P. Stratton \\ HRL Laboratories, LLC, 3011 Malibu Canyon Road, Malibu, CA, 90265-4799, USA
}

\begin{abstract}
We report on the development of a compensated, integrated, differential capacitive hydrogen sensor based on the lattice expansion of $\mathrm{LaNi}_{5-x} M_{x}$ thin films. Fabricated devices demonstrate a stable response to hydrogen as measured over 0.1 to $1 \mathrm{~atm}$. We have measured the intrinsic response time of $L a N i_{4.4} A l_{0.6}$ films as $\tau=10 \mathrm{~s}$. We have determined a Sieverts type dependence of the $\mathrm{LaNi}_{4.4} A l_{0.6}$ film response to hydrogen as a function of partial pressure. Additionally, we have shown the integrated sensor sensitivity to be better than 0.01 atm with the ability to detect hydrogen at pressures $>2 \mathrm{~atm}$.
\end{abstract}

\section{INTRODUCTION}

Recently there has been considerable focus on the emergence of a hydrogen economy, underscoring the need for low-cost wide dynamic range hydrogen sensors. While there are many commercially available sensors that respond in the range suitable for threshold sensors $(<4 \%$ hydrogen), many have issues associated with gas specificity and robustness. There are only a few examples of concentration sensors (4-100\% hydrogen) [1], and similar considerations apply. In this paper we report on a novel hydrogen sensor concept based on the lattice expansion of a metal hydride thin film, which has the potential to meet the demands of a broad range of sensing applications [2]. In this paper we will describe the thin film material characterization, and the wafer level fabrication and testing of a compensated thin film $\mathrm{LaNi}_{4.4} A l_{0.6}$ capacitive hydrogen sensor.

Alloys of the formula $L a N i_{5-x} M_{x}$ absorb hydrogen to form metal hydrides reversibly and exhibit a substantial lattice expansion as a consequence of absorption [3]. This physical property of $L a N i_{5-x} M_{x}$ alloys is used as the basis of a capacitive sensor. This sensor is ideally suited for hydrogen detection because the lattice expansion is specific to hydrogen and the temperature and pressure of hydriding can be tuned by varying the alloy composition. Thus, maximum sensitivity can be adjusted for specific operating conditions rendering a high dynamic range for detection of hydrogen.

\section{THIN FILM RESULTS}

We have prepared thin films of $\mathrm{LaNi}_{4.4} A l_{0.6}$ on Si (100) substrates by DC magnetron sputtering using elemental targets. We have measured the lattice expansion of these films using a tilted plate capacitance dilatometer [4] in a temperaturecontrolled oven, equipped with $\mathrm{N}_{2}$ and $\mathrm{H}_{2}$ gas handling capability. An expansion of the lattice is measured as a change in the capacitance gap of the dilatometer. Figure 1 shows a response time of $\tau=10$ s for a 2 m-thick $L a N i_{44} A l_{0.6}$ film on exposure to $0.8 \mathrm{~atm}$ of hydrogen, as measured at $\mathrm{T}=103^{\circ} \mathrm{C}$. The capacitance change of a bare silicon wafer on exposure to 0.8 atm hydrogen is plotted for reference. In this case, hydrogen does not adsorb on silicon or absorb into the silicon lattice, hence no expansion occurs and the measured capacitance change is due to the dielectric constant change of vacuum to hydrogen only.

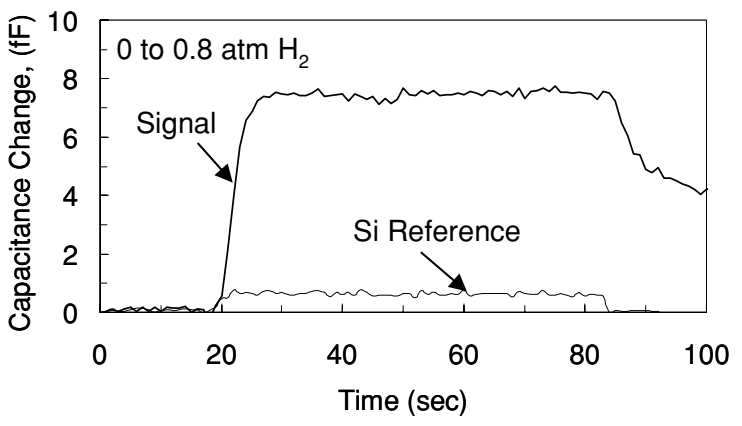

Figure 1. Response time of $2 \mathrm{~m}$ thick $\mathrm{LaNi}_{4.4} \mathrm{Al}_{0.6}$ film to $0.8 \mathrm{~atm}$ hydrogen showing $\tau_{90}=10 \mathrm{~s}$ at $T=103^{\circ} \mathrm{C}$. Silicon substrate response is shown for dielectric constant change only. Measurement integration time $=0.03$ seconds.

For sensor calibration, we have also measured the steady state response of films as a function of partial pressure of hydrogen. The thin films exhibit a Sieverts type dependence as shown in Figure 2, $\left(\Delta \mathrm{C} \alpha \mathrm{P}^{1 / 2}\right)$. Therefore the films have a larger fractional response at lower pressures indicating the potential for threshold sensor applications. Films have been tested from 0.1 to $3.0 \mathrm{~atm}$ of hydrogen at temperatures of 70 to $120^{\circ} \mathrm{C}$.

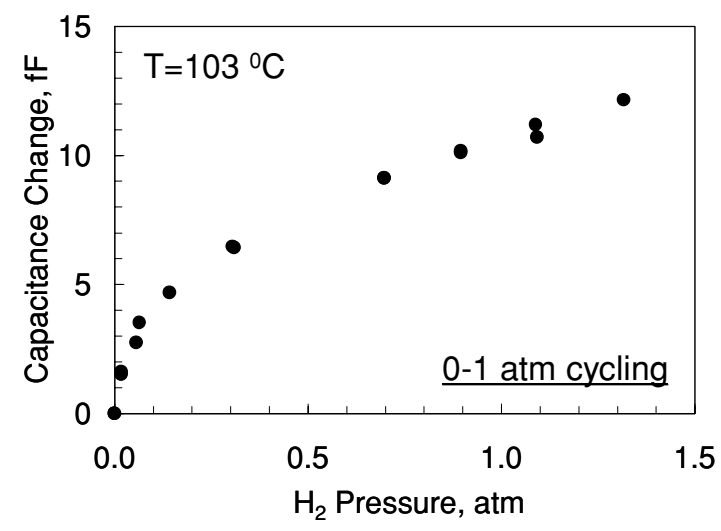

Figure 2. Sieverts type dependence of steady state response of $4 \mathrm{mLaNi}_{4.4} \mathrm{Al}_{0.6}$ film to hydrogen observed over 0 to $>1 \mathrm{~atm}$. 


\section{INTEGRATED DEVICE RESULTS}

We have successfully implemented these films in a compact differential device, which is designed to compensate for the effect of extraneous gases and thermal effects on the capacitance measurement. Our two-wafer process and the completed device are shown schematically in Figure 3. Two capacitors, signal and reference, are formed in close proximity. Gas delivery channels supply the same gas flow and mixture to both signal and reference capacitors in the sensing region so as to allow compensation for thermal and dielectric effects, other than the response to hydrogen.

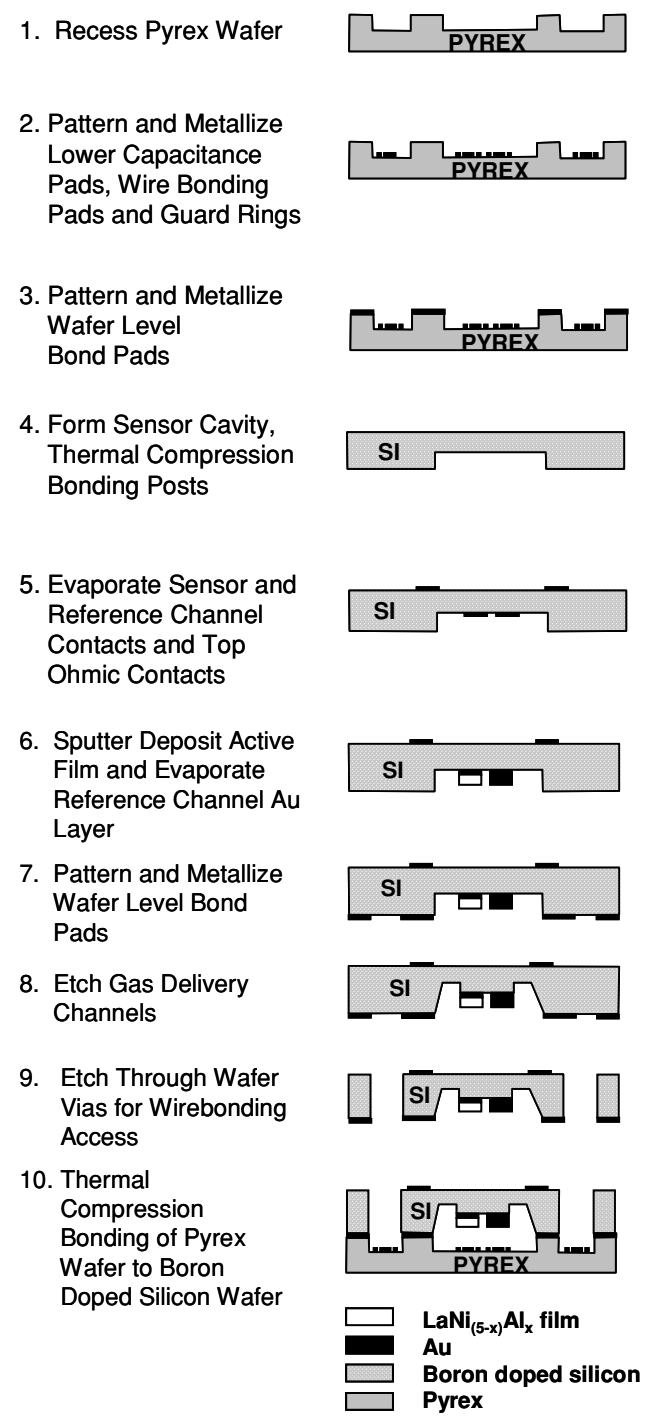

Figure 3. Hydrogen sensor two-wafer fabrication process. Active film areas are: $200 \mathrm{~m}^{2}, 500 \mathrm{~m}^{2}$ and $1000 \mathrm{~m}^{2}$.

Figure 4 shows the differential response of a $500 \mathrm{~m}^{2}$ integrated device to cycling of hydrogen between vacuum and 1 atm, illustrating a burn in of approximately 12 cycles at $\mathrm{T}=72^{\circ} \mathrm{C}$. The differential signal is the signal capacitance minus the reference capacitance. The negative abscissa values reflect that the reference capacitance is slightly higher than that of the signal. An initial cycle of $\mathrm{N}_{2}$ is used as an inert calibration gas to confirm that the lattice expansion occurs in the presence of hydrogen only. Figure 5 demonstrates that sensor sensitivity is better than $0.01 \mathrm{~atm}$ hydrogen at $\mathrm{T}=90{ }^{\circ} \mathrm{C}$, based on the signal to noise of the differential capacitance.

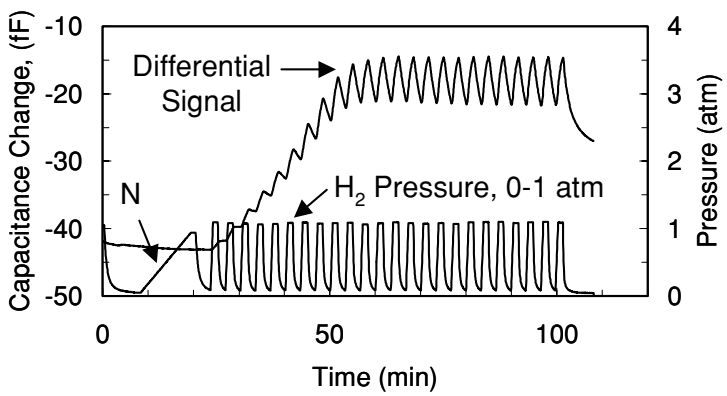

Figure 4. Response of $500 \mathrm{~m}^{2}$ integrated sensor to 0-1 atm of hydrogen at $T=72^{\circ} \mathrm{C}$. Burn-in $=12$ cycles.

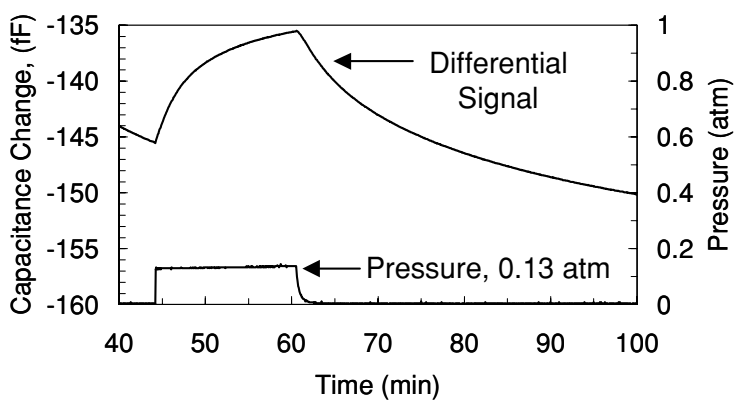

Figure 5. Differential response of $500 \mathrm{~m}^{2}$ integrated sensor to $0.13 \mathrm{~atm}$ of hydrogen at $\mathrm{T}=90^{\circ} \mathrm{C}$.

\section{SUMMARY}

In summary, we have demonstrated the feasibility of integrating a differential capacitive hydrogen sensor based on the lattice expansion of $\operatorname{LaNi}_{5-x} M_{x}$ thin films. The devices demonstrate a stable response to hydrogen as measured over 0.1 to $1 \mathrm{~atm}$. Integrated devices have a burn-in of typically 12 cycles of hydrogen after which the amplitude of the response stabilizes. We have measured the intrinsic response time of $\mathrm{LaNi}_{4.4} A l_{0.6}$ films as $\tau=10 \mathrm{~s}$, and it is expected that the devices will also demonstrate a $\tau=10 \mathrm{~s}$ in the absence of a process contamination. We have determined a Sieverts type dependence of the $L a N i_{4.4} A l_{0.6}$ film response to hydrogen as a function of partial pressure, indicating the potential for threshold sensor applications. We have demonstrated an integrated sensor sensitivity better than $0.01 \mathrm{~atm}$ and tested films over 0 - 2 atm of hydrogen partial pressure with stable response.

\section{ACKNOWLEDGMENTS}

This work was supported by General Motors - FCA division.

\section{REFERENCES}

[1] Baselt D R, el al, 2003, Sensors and Actuators B 88 120; see also www.h2scan.com.

[2] Zinck J J and Kirby D J, 2003, Thin film metal hydride hydrogen sensor, U. S. Patent \# 6,539,774

[3] Gupta M and Schlapbach L, 1988, Hydrogen in Intermetallic Compound I, Topics in Applied Physics, 63182 (Springer-Verlag)

[4] Genossar J and Steinitz M, 1990, Review Sci. Instrum., 61 2469. 